\title{
BMI open A randomised controlled trial comparing surgical intervention rates between two protocols for the management of asymptomatic adnexal tumours in postmenopausal women
}

\author{
Natalie Nunes, ${ }^{1}$ Xulin Foo, ${ }^{1}$ Martin Widschwendter, ${ }^{2}$ Davor Jurkovic, ${ }^{1}$
}

To cite: Nunes N, Foo X, Widschwendter $\mathrm{M}$, et al. A randomised controlled trial comparing surgical intervention rates between two protocols for the management of asymptomatic adnexal tumours in postmenopausal women. BMJ Open 2012;2: e002248. doi:10.1136/ bmjopen-2012-002248

- Prepublication history and additional material for this paper are available online. To view these files please visit the journal online (http://dx.doi.org/10.1136/ bmjopen-2012-002248).

Received 22 October 2012 Revised 22 October 2012 Accepted 30 October 2012

This final article is available for use under the terms of the Creative Commons Attribution Non-Commercial 2.0 Licence; see http://bmjopen.bmj.com

For numbered affiliations see end of article.

Correspondence to Davor Jurkovic; davor.jurkovic@nhs.net

\section{ABSTRACT}

Introduction: Detection of asymptomatic adnexal tumours in postmenopausal women has increased due to wider use of diagnostic ultrasound and imaging quality improvements. Reliable methods to differentiate between benign and malignant tumours are required to avoid delays in treating ovarian cancer and to prevent unnecessary interventions for benign lesions. In the UK, the Royal College of Obstetricians and Gynaecologists has issued guidance for the management of adnexal cysts in postmenopausal women, which is considered standard in routine clinical practice. The protocol utilises the Risk of Malignancy Index to assess the risk of adnexal lesion being malignant. This protocol has a relatively high intervention rate in order to avoid a delay in a cancer diagnosis. The Simple Rules Protocol designed by International Ovarian Tumour Analysis Group reports a low false-positive rate in the diagnosis of ovarian cancer without a loss of sensitivity and therefore has the potential to reduce unnecessary interventions in asymptomatic postmenopausal women with benign cysts.

Methods and analysis: 140 postmenopausal women aged 40-80, with incidentally detected adnexal tumours on ultrasound scan will be recruited to this study. They will be randomly allocated, to be assessed and managed according to either of the two protocols under investigation. In both arms of the study the tumours will be classified into three groups: high, intermediate or low risk of malignancy. Women with high risk of malignancy will be referred for management in a tertiary cancer centre, women with low-risk tumours will be managed expectantly, while those with intermediate risk findings have surgery in their local hospital units. Analysis will be on an intention-to-treat basis.

Ethics and dissemination: Research ethical approval was granted by the North London Research Ethical Committee 2 (10/H0724/48). Trial results will be published according to the CONSORT statement.

Trial registration number: Registration at http:// www.controlled-trials.com/ISRCTN89034131/. ISRCTN89034131

\section{ARTICLE SUMMARY}

Article focus

- The primary objective is to assess the differences in surgical intervention rates between two different protocols for the management of incidentally detected adnexal tumours in postmenopausal women.

- Assessment of adnexal tumours using the Risk of Malignancy Index (RMI) calculation alongside the guidance from the Royal College of Obstetricians and Gynaecologists (RCOG) as compared with the Simple Rules as designed by the International Ovarian Tumour Analysis Group.

Key messages

- Most asymptomatic adnexal tumours detected on ultrasound scan are benign. In these women, an operation to remove the cyst is unlikely to be beneficial and may do harm.

- A test, which can better discriminate between benign and malignant tumours will afford women without a malignancy to decline surgery.

Strengths and limitations of this study

- This is the first randomised controlled trial to assess the RMI and the RCOG protocol against the Simple Rules protocol for the management of asymptomatic adnexal tumours in postmenopausal women.

- It is a single centre study, which can affect applicability of the results in other units.

- Both assessment and management protocols have high sensitivity rates with low false-negative rates.

\section{INTRODUCTION}

Wider and more liberal use of diagnostic ultrasound in gynaecological clinics has resulted in the detection of adnexal tumours in a large number of asymptomatic postmenopausal women. There has also been an increase in the use of other imaging modalities such at CT and MRI to assess a variety of 
medical complaints resulting in incidental detection of adnexal tumours. Any adnexal cyst detected in menopause has a potential of being malignant.

In the UK, the Royal College of Obstetricians and Gynaecologists (RCOG) has issued a guideline for the management of cysts in postmenopausal women, which is widely used in the routine clinical practice. ${ }^{1}$ According to this protocol all adnexal cysts/tumours are categorised as high, intermediate and low risk of being malignant. This categorisation is based on the Risk of Malignancy Index (RMI) calculation ${ }^{2}$ This model calculates a score which is the product of the value of the CA $125(\mathrm{U} / \mathrm{ml})$, a score for menopausal status (1 if premenopausal and 3 if postmenopausal) and a greyscale ultrasound score of 0,1 or 3 where 1 score is given each for bilaterality, ascites, multilocular, solid areas and intra-abdominal metastases. In the original study by Jacobs, a score of $>200$ gave a sensitivity of $85 \%$ and a specificity of $97 \%$ for the diagnosis of ovarian cancer. A recent systematic review of 16 studies assessing the diagnostic performance of RMI showed that the test had an overall sensitivity of $78 \%$ (95\% CI $71 \%$ to $85 \%$ ) and a specificity of $87 \%(95 \%$ CI $83 \%$ to $91 \%){ }^{3}$

The RMI has the advantage of being simple and widely used. Its disadvantage though is that it includes a blood test for CA125, which adds to the cost. In addition the diagnosis is delayed until the result of blood test is available. The absolute value of the RMI and the level of CA125 in serum are used to determine the management plan. Simple unilateral adnexal cysts of $<5 \mathrm{~cm}$ with an RMI of $<25$ and where the CA 125 is $<30 \mu / \mathrm{ml}$ are considered low risk. For cysts with an $\mathrm{RMI}<25$ but where the CA $125 \geq 30 \mu / \mathrm{ml}$, the cyst is $\geq 5 \mathrm{~cm}$ or the cyst has septations or solid areas then these are considered of intermediate risk. Those with an RMI of 25-250 are also considered to be of indeterminate risk. Adnexal tumours with an RMI $>250$ are classified as high risk. ${ }^{1}$ (Flowchart) As the main aim of this protocol is to minimise the risk of delaying interventions in women with ovarian cancer, the overall intervention rates are relatively high and many women with benign lesions are treated by surgery.

In recent years, the International Ovarian Tumour Analysis (IOTA) collaboration has developed several novel diagnostic models with the aim of improving noninvasive diagnosis of ovarian cancer. In 2008, the collaboration proposed use of 'Simple Rules' to assess tumours. ${ }^{4}$ The 'Simple Rules' are based on a structured approach to morphological analysis of ovarian tumours on ultrasound scan. It enables discrimination between benign and malignant lesions without the need to measure tumour markers.

Ultrasound scan 'Simple Rules' use 10 rules to assess adnexal tumours. There are five rules to predict malignancy (M-rules): (1) irregular solid tumour; (2) presence of ascites; (3) at least four papillary structures; (4) irregular multilocular-solid tumour with a largest diameter of at least $100 \mathrm{~mm}$ and (5) very high colour content on colour Doppler examination (colour score 4). There are five rules to suggest a benign tumour (B-rules): (1) unilocular cyst; (2) the presence of solid components where the largest solid component is $<7 \mathrm{~mm}$ in largest diameter; (3) acoustic shadows; (4) smooth multilocular tumour less than $100 \mathrm{~mm}$ in largest diameter and (5) no detectable blood flow on Doppler examination (colour score 1$)$.

If one or more M-rules apply in the absence of a B-rule, the mass is classified as malignant. If one or more B-rules apply in the absence of an M-rule, the mass is classified as benign. If no rule applies or both $\mathrm{M}$ and $\mathrm{B}$ rules apply, the mass cannot be classified and defined as indeterminate.

These 10 rules were applicable to $76 \%(937 / 1233)$ of all tumours in the study, where they resulted in a sensitivity of $93 \%$, specificity of $90 \%$, positive likelihood ratio $(\mathrm{LR}+)$ of 9.45 and negative likelihood ratio (LR-) of 0.08 . When prospectively tested the rules were applicable in $76 \%(386 / 507)$ of the tumours, where they had a sensitivity of $95 \%(106 / 112)$, a specificity of $91 \%$ (249/274), LR+ of 10.37 and LR- of 0.06. The 'Simple Rules' were subsequently tested prospectively. ${ }^{5}$ The rules were applicable in $77 \%$ of the 1938 women with a sensitivity of $92 \%$ (95\% CI $89 \%$ to $94 \%$ ) and specificity of 96\% (94\% to $97 \%)$.

The Simple Rules Model is particularly suitable for the use in standard clinical practice and, similar to the RMI, can be modified to divide the cysts into high, intermediate and low-risk categories. There is some evidence, albeit limited, that the Simple Rules model may be superior to the RMI in terms of sensitivity and specificity. ${ }^{2} 56$ Successful use of Simple Rules require more skilled ultrasound operators, but there in no need for additional blood tests and the results are available instantly.

\section{METHODS AND ANALYSIS}

The aim of this study was to assess the difference in the intervention rates between two different protocols for the management of incidentally detected adnexal tumours in postmenopausal women.

This is a prospective randomised controlled trial that will take place at the University College London Hospital gynaecology clinic. Women will be randomised into two groups. The first group will be assessed and managed in accordance with the current RCOG protocol for the management of cysts in postmenopausal women. ${ }^{1}$ The second group of women will be assessed and managed using the 'Simple Rules' protocol. ${ }^{4}$

\section{Inclusion criteria}

All postmenopausal women aged 40-80 who are referred for an asymptomatic adnexal tumour or those found to have one at the time of their visit to the gynaecology unit will be invited to take a part of the study. Postmenopausal women are defined as those who have had 1 year of spontaneous amenorrhoea at or above the age of 40 where no illness or medication may have 
caused the amenorrhoea or those at or above the age of 50 who have had a hysterectomy. Women will be judged to be asymptomatic if they did not present with pain localised to the area of the tumour or the lower pelvis.

Women who present with pelvic pain that could be attributed to the tumour and those who are younger than 40 or older than 80 years of age will be excluded. Women who are unable to consent and those with simple unilateral unilocular cysts $<2 \mathrm{~cm}$ in size will also be excluded as the RCOG guideline stipulates these women do not require follow-up.

\section{Interventions}

All women will have an initial ultrasound scan. All examinations will be performed by level II ultrasound operators, who have been fully trained in the assessment of adnexal tumours using both the RMI and 'Simple Rules' approach. Those randomised to the RCOG/RMI group will also have a blood test taken for the measurement of serum CA125. The tumours will be classified as low, intermediate and high risk as previously described (flowchart). In the Simple Rules group, adnexal tumours will be classified as low risk if one or more B-rules apply in the absence of an M-rule. If one or more M-rules apply in the absence of a B-rule, the mass will be classified as high risk. If no rules apply or if both $\mathrm{M}$ and $\mathrm{B}$ rules apply, the tumour will be classified as intermediate risk.

Women with tumours classified as high risk of malignancy by either protocol will be referred to the Gynaecological Oncology team. Tumours found to be of intermediate risk will be offered surgery by the general gynaecology team. All asymptomatic women diagnosed with low-risk tumours will be managed conservatively and have 4 monthly scans for 1 year observing for any changes in the tumour size or morphology. Should the characteristics of the tumour change prompting its reclassification into intermediate or high-risk category the management plan will be modified accordingly. Following conclusion of the study all women who did not require an intervention will be offered yearly follow-up ultrasound scans for a further 4 years.

\section{Randomisation}

A statistician using a Stata V.12.1 (Stata Corp., College Station, Texas, USA) will generate the blocked randomisation list with varying block sizes. The randomisation numbers will be placed in sealed, opaque, numbered envelopes and kept in a box locked in a filing cabinet. This randomisation ensures allocation concealment. When a patient consents, a clinic nurse who is not part of the research team will access the next envelope and give it to the recruiting doctor.

\section{Outcome measures}

The primary outcome is the rate of surgical intervention. Secondary outcomes include the number of delayed diagnoses of ovarian cancer, number of staging surgical procedures and number of surgical complications.

\section{Data collection and statistical analysis}

The data are collected on a predesigned proforma, which is then filed. This is transferred to a Microsoft Excel database in preparation for analysis. A $\chi^{2}$ test or Fisher's exact test will be used will be used to assess significant differences in the intervention rates between the two management protocols.

Analysis will be on an intention-to-treat basis of all patients who fit the inclusion criteria. The researchers will do the analysis with the statistician at the midpoint and at the end.

\section{Pilot study and sample size}

A preliminary prospective audit of 67 patients was done. These patients were aged 47-90 years. Using RMI/ RCOG group protocol $41 / 67(61.2 \%$ (95\% CI $48.5 \%$ to $72.9 \%)$ ) were classified as requiring a surgical intervention compared to $5 / 67(7.5 \%$ (95\% CI $2.5 \%$ to $16.6 \%)$ ) when the 'Simple Rules' protocol was used. The women with the final diagnosis of ovarian cancer were classified as high $(\mathrm{n}=1)$ or intermediate $(\mathrm{n}=1)$ risk by both protocols and they both had surgery performed by the gynaecological oncology team. We will assume that some patients offered conservative management will choose to still have surgery and that some patients who are offered surgery will choose not have an operation.

A significant improvement of surgical rates is considered to be a $50 \%$ reduction without a significant difference in the number of women with a delayed diagnosis of ovarian cancer. Therefore, if use of the 'Simple Rules' halves the intervention rate from $60 \%$ to $30 \%$ and assuming a 5\% significance level and 90\% power, 63 patients would be required in each group. Allowing for a $10 \%$ drop-out rate, 140 patients will be needed. The sample size was calculated by use of Stata V.12.1 (Stata Corp.) as provided by evidence from Machin et al. ${ }^{7}$ There was a $3 \%$ incidence of ovarian cancer in our pilot study population so we would expect to find four cancer diagnoses in the study population.

\section{DISCUSSION}

Most asymptomatic adnexal tumours detected on ultrasound scan are benign. In these women, an operation to remove the cyst is unlikely to be beneficial and may do harm.

By avoiding surgery women are not exposed to surgical and anaesthetic complications and their care is likely to be more cost effective. Recent trials have shown that unnecessary interventions in women with benign adnexal lesions lead to significant morbidity and mortality, which offsets the potential benefits of screening for ovarian cancers. ${ }^{8}$ Postmenopausal women are also more likely to suffer from chronic medical problems such as diabetes and high blood pressure, which increase operative and anaesthetic risks. Women with presumed benign cysts will be observed over the following year in 3-4 monthly intervals in order to detect any change in 
appearance or increase in size, which would be suspicious of malignancy. Any suspicion of malignant change would trigger a surgical intervention.

We will record sensitivities of both protocols for the detection of ovarian cancer. Although our audit showed that all cases of ovarian malignancies were correctly classified by both protocols, it is important to confirm this observation in a larger prospective study. We do expect, however, that the specificity of the 'Simple Rules' protocol will be higher, which should translate into lower intervention rates.

If the study shows that the use of the 'Simple Rules' and conservative management could substantially reduce intervention rates without increasing the risk of delaying the diagnosis of ovarian cancer this could have a positive impact on clinical practice, increase patients' safety and result in significant savings for the health services.

\section{Ethics and dissemination}

Approval for this study was obtained from the North London Research Ethical Committee 2 (North REC 2), London, UK (10/H0724/48). Written informed consent is obtained from each patient fulfilling the inclusion criteria before randomisation. Women who refuse participation are recorded. There is a small risk to patients of a delayed diagnosis of ovarian cancer and there are also the risks of surgery for those who are offered surgical intervention.

\section{Incident reporting}

Adverse events will be recorded from recruitment to the 1-year-scan date for those who have conservative management or to 3 months postoperatively for those who have surgery. The chief investigator will be responsible for the reporting of all serious adverse events or suspected unexpected serious adverse reactions immediately or as soon as the trial personnel become aware of an event. The chief investigator will report all fatal or life-threatening events as soon as possible to the trial coordinating centre. This will be done not later than 7 days after the chief investigator is first aware of the event. All events which are not fatal or life-threatening will also reported as soon as possible and not later than 15 days after the chief investigator is first aware of the reaction. The research and ethics committee also require a report of all SAEs and SUSARs within
15 days of the chief investigator being made aware. The principal investigator will also follow all SAEs and SUSARs through to outcome.

\section{Author affiliations}

${ }^{1}$ Gynaecological Diagnostic Outpatient Treatment Unit, University College Hospital, London, UK

${ }^{2}$ Department of Women's Cancer, University College London, Elizabeth Garrett Anderson, Institute for Women's Health, London, UK

Acknowledgements I would like to acknowledge Mr Paul Bassett as the statistician who did the sample calculation and generated the randomisation list.

Contributors NN and DJ conceived the idea and contributed to the design along with MW. XF contributed to design and NN with XF are responsible for data acquisition. NN and DJ prepared the initial article draft and circulated it to the other authors XF and MW until all authors approved the final article for submission.

Funding This research received no specific grant from any funding agency in the public, commercial or not-for-profit sectors.

Competing interests None.

Ethics approval North London Research Ethical Committee 2 (North REC 2).

Provenance and peer review Not commissioned; externally peer reviewed

Data sharing statement No additional data are available.

\section{REFERENCES}

1. RCOG Ovarian cysts in postmenopausal women Guideline No. 34 October 2003.

2. Jacobs I, Oram D, Fairbanks J, et al. A risk of malignancy index incorporating CA125, ultrasound and menopausal status for the accurate preoperative diagnosis of ovarian cancer. $\mathrm{Br} J$ Obstet Gynaecol 1990;97:922-9.

3. Geomini P, Kruitwagen R, Bremer GL, et al. The accuracy of risk scores in predicting ovarian malignancy: a systematic review. Obstet Gynecol 2009;113(2 Pt 1):384-94.

4. Timmerman D, Testa AC, Bourne T, et al. Simple ultrasound-based rules for the diagnosis of ovarian cancer. Ultrasound Obstet Gynecol 2008;31:681-90.

5. Timmerman D, Ameye L, Fischerova D, et al. Simple ultrasound rules to distinguish between benign and malignant adnexal masses prior to surgery: a prospective validation by the IOTA group. BMJ 2010;341:c6839.

6. Di Legge A, Testa AC, Ameye $L$, et al. Lesion size affects diagnostic performance of IOTA logistic regression models, IOTA simple rules and risk of malignancy index in discriminating between benign and malignant adnexal masses. Ultrasound Obstet Gynecol 2012;40:345-54.

7. Machin D, Campbell MJ, Tan SB, et al. Sample size tables for clinical studies. Comparing paired groups of binary, ordered categorical and continuous outcomes. Oxford, UK: Wiley-Blackwell, Third 2009, 69-83.

8. Buys SS, Partridge E, Black A, et al. Effect of screening on ovarian cancer mortality: the prostate, lung, colorectal and ovarian (PLCO) cancer screening randomized controlled trial. JAMA 2011;305:2295-303. 